\title{
Medical Equipment Comprehensive Management System Based on Cloud Computing and Internet of Things
}

\author{
Lin Yao, Danmei Shang $(D$, Hui Zhao, and Shuyu Hu \\ College of Public Basic Sciences, Jinzhou Medical University, Jinzhou, Liaoning 121001, China \\ Correspondence should be addressed to Danmei Shang; wzhsdm@jzmu.edu.cn
}

Received 19 November 2020; Revised 17 December 2020; Accepted 18 February 2021; Published 3 March 2021

Academic Editor: Yang Gao

Copyright ( 92021 Lin Yao et al. This is an open access article distributed under the Creative Commons Attribution License, which permits unrestricted use, distribution, and reproduction in any medium, provided the original work is properly cited.

\begin{abstract}
The continuous progress in modern medicine is not only the level of medical technology, but also various high-tech medical auxiliary equipment. With the rapid development of hospital information construction, medical equipment plays a very important role in the diagnosis, treatment, and prognosis observation of the disease. However, the continuous growth of the types and quantity of medical equipment has caused considerable difficulties in the management of hospital equipment. In order to improve the efficiency of medical equipment management in hospital, based on cloud computing and the Internet of Things, this paper develops a comprehensive management system of medical equipment and uses the improved particle swarm optimization algorithm and chicken swarm algorithm to help the system reasonably achieve dynamic task scheduling. The purpose of this paper is to develop a comprehensive intelligent management system to master the procurement, maintenance, and use of all medical equipment in the hospital, so as to maximize the scientific management of medical equipment in the hospital. Scientific Management. It is very necessary to develop a preventive maintenance plan for medical equipment. From the experimental data, it can be seen that when the system simultaneously accesses 100 simulated users online, the corresponding time for submitting the equipment maintenance application form is $1228 \mathrm{~ms}$, and the accuracy rate is $99.8 \%$. When there are 1000 simulated online users, the corresponding time for submitting the equipment maintenance application form is $5123 \mathrm{~ms}$, and the correct rate is $99.4 \%$. On the whole, the medical equipment management information system has excellent performance in stress testing. It not only predicts the initial performance requirements, but also provides a large amount of data support for equipment management and maintenance.
\end{abstract}

\section{Introduction}

In recent years, the rapid development of medical technology has made the average medical level of the entire society continue to rise, and various advanced medical equipment have been developed and put on the market [1]. As the number and types of medical equipment purchased by hospitals increase, the maintenance and maintenance requirements for medical equipment are also higher. How to manage and maintain these pieces of medical equipment with a large number of brands and advanced science and technology and give full play to their role in medical treatment is a very important issue for the hospital department managers and maintenance engineers and technicians. With the continuous expansion of medical equipment in hospitals, the speed of equipment upgrades and updates has accelerated, and the information items for maintenance management have become more and more complicated. The requirements for equipment maintenance management and information retrieval have become higher and higher.

In foreign countries, many scholars have conducted large-scale research and discussion on the management of medical equipment. Amerieon et al. conducted a qualitative study on the factors affecting the maintenance and management of medical equipment in military hospitals. He used the framework analysis method to investigate the managers and medical equipment experts of a military area hospital in a targeted manner. Semistructured interviews are used for data analysis and descriptive statistics are used to rank the 
frequency of many factors affecting medical equipment maintenance management. From the experimental data, equipment management training has a very important proportion. Of course, due to the limited sample data, they have considered the convincing power of the results to a certain extent [2]. Ulickey conducted research on rich cases of integrated facility management systems. Networking and the innovation of digital control systems have enabled the integration of different control strategies in the past. These strategies are not only suitable for building systems, but also in the field of medical management. Scientific innovation provides a more powerful mathematical basis for the rational use of various equipment in the hospital. In the future, people should focus on how to correctly understand these data and promote the intelligent scheduling effect of the system [3].

With the development of emerging science and technology, domestic hospitals have paid more and more attention to the maintenance and management of medical equipment. Qiang believes that advanced medical equipment is one of the important signs of modern hospital technology. Therefore, the hospital has the responsibility to establish an effective management model and manage the medical equipment to keep it in a good operating condition and ensure the safety of patients. He and his team used a variety of methods such as literature research, questionnaire survey, and data analysis to summarize the problems in the maintenance and management of hospital medical equipment, the development and characteristics of the maintenance management model, and the current situation at home and abroad [4]. In the research, he proposed that the use of advanced Internet technology to develop intelligent systems has a high feasibility in the management of medical equipment, but he did not prove its advantages in specific practical research [5].

Based on cloud computing and the Internet of Things technology, this paper has launched an in-depth study on the integrated management system of medical equipment. The research is mainly carried out from the following parts: first, this article describes the technologies and methods used in the system development, including cloud computing and task scheduling, IoT intelligent control system, particle swarm algorithm, and chicken swarm optimization algorithm. Then, this article starts with the network structure, software structure, development environment, database, and other aspects to develop a comprehensive medical equipment management system platform. Finally, this article simulates the effect of the system in practical applications and various possible problems from the perspective of the procurement, allocation, maintenance, and use of medical equipment.

\section{Technology Based on Medical Equipment Integrated Management System}

2.1. Cloud Computing and Task Scheduling. Cloud computing is an emerging technology model that pays on demand [6]. It can provide people with the resources they need in the shortest time, which is very convenient and fast [7].
Compared with traditional computer technology, the biggest difference of cloud computing is that it is based on virtualization technology [8], using the network as the carrier, and integrating large-scale and scalable computing, storage, data, applications, and other distributed computing resources for collaborative work (the supercomputing model) $[9,10]$.

In this environment of explosive growth in the amount of information, more and more industries are beginning to use various Internet emerging technologies to achieve intelligent system optimization and management, and the advancement of cloud computing technology provides a powerful way for large-scale task mobilization (technical support) [11]. In the medical field, the problem information involved is usually diverse and highly specialized. Taking medical equipment management as an example, if you want to develop a system that can realize intelligent management, you cannot do this without the support of cloud computing and the Internet of Things technology. Cloud computing task scheduling is mainly to study how to allocate resources for tasks submitted by users. In other words, it is to allocate multiple independent and diversified tasks to the large-scale virtual resources in the cloud, so as to satisfy all tasks with the highest efficiency (user needs) [12, 13].

Suppliers of cloud computing platforms pay most attention to the utilization of data resources, energy consumption, and the level of profit during the use of the platform; users pay attention to service quality and cost, no matter from which point of view, task scheduling is shared by people (an important stage of virtual resources) [14]. Its essence is the process of reasonably assigning tasks submitted by users to virtual resources. Therefore, the improvement of algorithm performance in task scheduling is the root of the whole problem $[15,16]$.

2.2. Internet of Things Intelligent Control System. The Internet of Things connects all items to the Internet through information sensing methods such as radio frequency identification, infrared induction, optical induction, and barcode scanning, so as to achieve information exchange and communication and achieve intelligent identification, positioning, tracking, monitoring, and management $[17,18]$. Figure 1 shows a diagram of the IoT system architecture.

As shown in Figure 1, the basic system of the Internet of Things can be divided into three parts: perception layer, network layer, and application layer. The perception layer is the foundation of the application and development of the Internet of Things. It is composed of various devices with perception capabilities. It mainly realizes information collection, object recognition, and other perception functions and has the ability to fully perceive the Internet of Things; the network layer integrates various communication networks and the Internet is used to build a collaboratively aware network to optimize and improve the application characteristics of the Internet of Things; the application layer is the fundamental purpose of the development of the Internet of Things, and its role is to combine the industry's informatization needs with the Internet of Things 

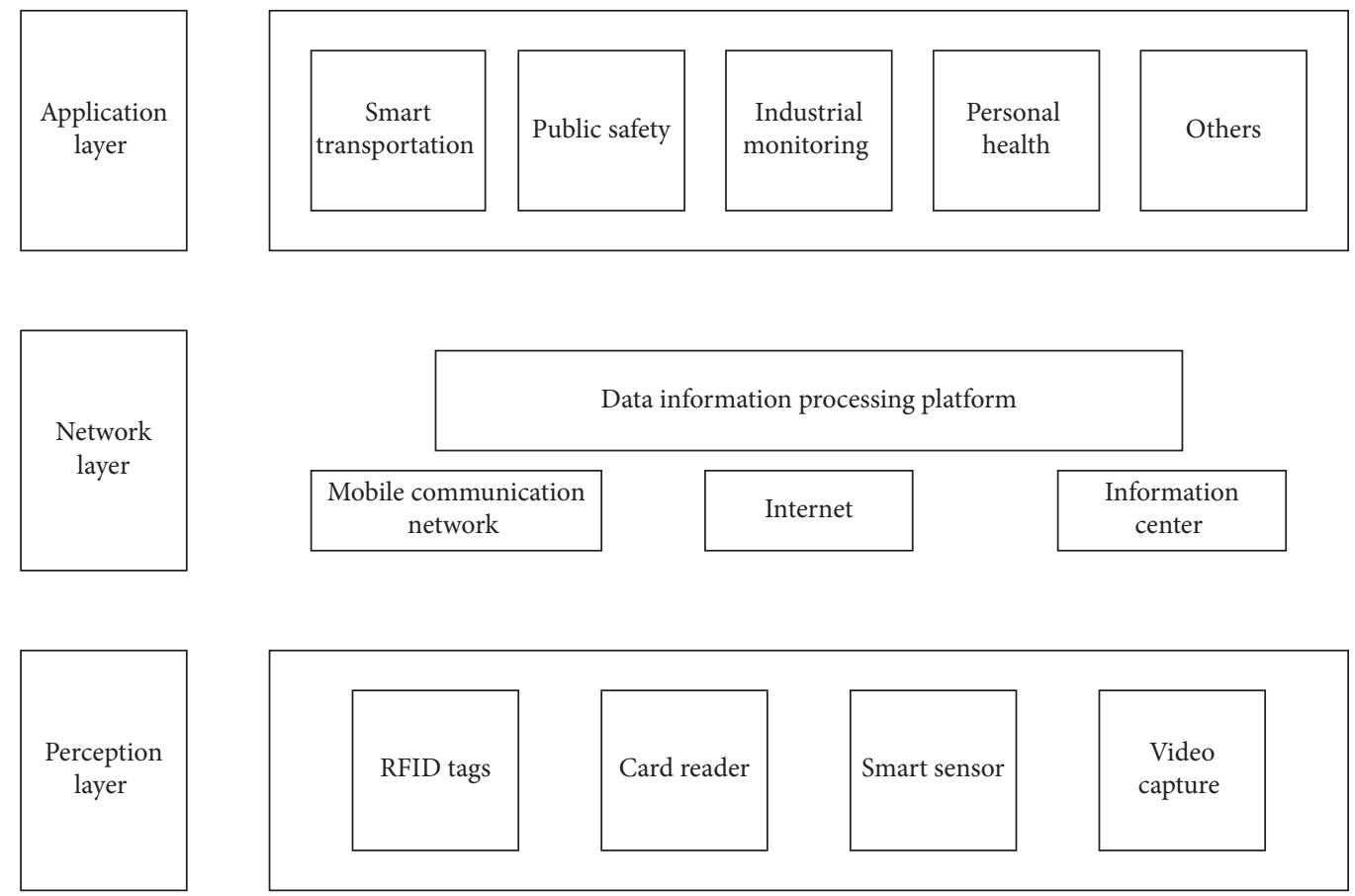

FIgURE 1: IoT system architecture diagram.

technology to provide different users with, for example, applications in the fields of transportation, security, environment, home furnishing, industry, and military and national defense [19].

\subsection{Particle Swarm Algorithm}

2.3.1. Basic Particle Swarm Algorithm. In order to realize the comprehensive management of medical equipment in the system based on cloud computing and the Internet of Things, intelligent algorithms need to be optimized to achieve reasonable task scheduling [20]. The essence of particle swarm optimization (PSO) is to focus on the appropriate value of the target search space and to judge the pros and cons of all individuals in between [21]. Each individual is like an example of movement in the group space and contains two parameters: speed $v i$ and position $x i$. Set the total number and dimension of examples in the population as $m, n$, respectively, $\omega$ is the inertia weight, and $c$ is the acceleration coefficient, that is, the learning factor. In the process of population global optimization, the speed and position of the example satisfy the formula

$$
\begin{aligned}
& v_{i d}=\omega v_{i d}+c_{1} r_{1}\left(p_{i d}-x_{i d}\right)+c_{2} r_{2}\left(p_{g d}-x_{i d}\right), \\
& x_{i d}=x_{i d}+v_{i d} .
\end{aligned}
$$

In the iterative process, the particle swarm optimization algorithm only transmits the information of the optimal solution to other particles and completes the search faster through the particles. This is a significant advantage of particle swarm optimization algorithm, which can minimize the waste of time and improve the search efficiency [22]. Coupled with fewer parameters involved in the particle swarm algorithm, it is easier to implement in actual work. On the other hand, the particle swarm algorithm also has certain shortcomings. For example, the speed of particles cannot be dynamically adjusted, and it is easy to fall into a local optimal solution, which leads to low convergence accuracy and inability to solve discrete problems and combinatorial optimization (question) [23]. In order to improve these situations, the particle swarm algorithm needs to be improved appropriately.

2.3.2. Improved Particle Swarm Algorithm. In order to solve the problem that the particle swarm algorithm is easy to fall into the local optimal solution and affect the task completion time, this paper combines the adaptive inertial weight and the random factor to better balance the local search and the global search, avoid falling into the local optimal solution, improve global optimization ability, and then obtain a task scheduling scheme with shorter task completion time and lower cost [24].

To apply the particle swarm algorithm to the cloud computing task scheduling problem, the particles need to be coded. If there are $k$ tasks and $t$ resources, each task corresponds to 1 resource. Defining matrix time $[i, j]$, the execution time for completing task $i$ in resource $j$ is

$$
\operatorname{Time}(j)=\sum_{i=t}^{n} \operatorname{time}[i, j] \quad(1 \leq j \leq t) .
$$

Among them, $n$ represents the total number of tasks executed in the resource and the total time to complete all tasks in the system and the fitness function satisfy the formula 


$$
\begin{aligned}
\text { task time } & =\max (\operatorname{Time}(j)) \quad(1 \leq j \leq t), \\
\text { fitness } & =\frac{1}{(\text { task time }+ \text { cost })} .
\end{aligned}
$$

This experiment will compare the total completion time and cost of all tasks with other algorithms, so the objective functions are defined as

$$
\begin{aligned}
\text { task time } & =\text { finish time }- \text { start time } \\
\text { cost } & =\text { time cost }+ \text { debt. }
\end{aligned}
$$

$P_{s}(t)$ is the success rate of the particle swarm iteration, indicating the proportion of particles in the particle swarm that are better positioned in this iteration than the last time. The calculation method of the success rate is as follows:

$$
P_{s}(t)=\frac{\sum_{i=1}^{n} S(i, t)}{n} \text {. }
$$

Among them, $\sum_{i=1}^{n} S(i, t)$ represents the sum of the success values of all particles, and $\omega(t)$ is the inertia weight during iteration, used to adjust the particle velocity during iteration:

$$
\omega(t)=\left(\omega_{\max }-\omega_{\min }\right) P_{s}(t)+\omega_{\min } .
$$

Suppose the joint distribution function of random variable $x, y$ is $H: R^{2} \longrightarrow[0,1]$, and its marginal distribution function is $F x, F y$, respectively. The theorem conforms to the formula

$$
\begin{aligned}
& H\left(x_{1}, x_{2}\right)=C\left(F_{x}\left(x_{1}\right), F_{y}\left(x_{2}\right)\right), \\
& C\left(u_{1}, u_{2}\right)=H\left(F_{x}^{-1}\left(x_{1}\right), F_{y}^{-1}\left(x_{2}\right)\right) .
\end{aligned}
$$

For any $(u, v) \in[0,1]^{2}$, the binary Copula function can be defined as

$$
C_{p}(u, v)=\varphi_{p}\left(\varphi^{-1}(u), \varphi^{-1}(v)\right) .
$$

Among them, $\Phi$ is the standard normal distribution function and $\Phi p$ is the joint distribution function of twodimensional normal variables [25]. Assuming that the random variable and the random factor meet a certain correlation, we can define that they conform to

$$
H\left(r_{1}, r_{2}\right)=C\left(r_{1}, r_{2}\right) \text {. }
$$

By comparing the fitness of each particle with the global optimal value, the position state of the particle can be described more accurately [26]. By refining the particle state, a more accurate success value can be obtained, and the success rate of the particle can be further improved, thereby improving the adaptability of the inertia weight and effectively avoiding the particle from falling into the local optimum early in the optimization process $[27,28]$.

\subsection{Chicken Flock Optimization Algorithm}

2.4.1. Basic Chicken Optimization Algorithm. Chicken flock optimization algorithm (CSO) is a bionic algorithm generated around the hierarchy of chicken flocks [29]. By simulating the living habits and hierarchy of the entire chicken flock, the entire chicken flock is divided into several groups, and there is competition between groups, which effectively reduces the time for the algorithm to perform tasks and can solve problems more quickly; and there are three members of rooster, hen, and chicken in each group. Members of different species have following relationships and competition relationships, and members of the same species also have competitive relationships. This hierarchical behavior within the group can improve the algorithm's global search capability and the efficiency of finding the optimal solution, so that the algorithm can efficiently solve practical problems $[30,31]$.

Set the number of roosters, hens, chicks, and mother hens in the flock to be $N r, N h, N c$, and $N m$, respectively; then, the position update formula of the roosters, hens, and chicks satisfies

$$
\begin{aligned}
x_{i d}^{k+1}= & x_{i d}^{k} \cdot\left(1+\operatorname{randn}\left(0, \sigma^{2}\right)\right), \\
x_{i d}^{k+1}= & x_{i d}^{k}+C 1 \cdot \operatorname{randn} \cdot\left(x_{j_{1} d}^{k}-x_{i d}^{k}\right) \\
& +C 2 \cdot \operatorname{randn} \cdot\left(x_{j_{2} d}^{k}-x_{i d}^{k}\right), \\
x_{i d}^{k+1}= & x_{i d}^{k}+F \cdot\left(x_{m d}^{k}-x_{i d}^{k}\right) .
\end{aligned}
$$

The advantage of the chicken flock optimization algorithm is that multiple groups can greatly reduce the probability of the algorithm searching for the optimal position and reduce the task scheduling time, and there is a competitive relationship between various groups and individuals, which is beneficial to task allocation. For load balancing, improve the quality of service [32]. However, there are three types of individuals in the algorithm: rooster, hen, and chicken. According to the characteristics of the flock's activities, the leading rooster will inevitably affect the trajectory of the hen and the chicken [33]. If the position of the head chicken is not the optimal position, the algorithm will fall into a local optimum; it is difficult for the hens and chicks that follow to jump out at this time, so in order to preserve the advantages of the algorithm and apply it to task scheduling, the algorithm must be optimized [34].

\subsubsection{Improved Chicken Optimization Algorithm.} Considering that, in the chicken swarm optimization algorithm, the chicken will follow the hen's action, and the hen will follow the lead of the rooster, which will easily lead to the problem of local optimal solution in the calculation process [35]. Therefore, according to the characteristics of chaotic perturbation traversing individuals, perturbation is added to the population to reduce the premature phenomenon caused by blind following. The disturbance formula satisfies

$$
S_{k+1}=4 S_{k}\left(1-S_{k}\right), S_{k} \in(0,1) \text {. }
$$

The improved chicken position update formula satisfies

$$
x_{i d}^{k+1}=\omega x_{i d}^{k}+F \cdot\left(x_{m d}^{k}-x_{i d}^{k}\right) .
$$

Among them, $\omega$ represents the coefficient that the chicken learns by itself and $F$ represents the following coefficient of the chicken following the hen foraging. From the 
experimental results, the improved chicken flock optimization algorithm effectively reduces the local optimal problem caused by the chicken blindly following the hen.

\section{Experimental Research on Comprehensive Management System of Medical Equipment}

3.1. Experimental Background. The development of modern medicine needs the help of high-tech medical equipment. How to ensure the normal operation of these medical equipment and give full play to its maximum social and economic benefits is the following problem, and the core of this problem is how to do a good job in equipment management. This article aims to design a set of real-time information management system for the current state of medical equipment. For this reason, it is necessary to analyze the overall requirements of the system before the start of the experiment and to fully understand the functional requirements required by the current medical equipment management, in order to achieve more targeted system design.

3.2. Experimental System Module Design. This system is based on ASP.NET dynamic website technology and SQL Server database. According to the overall function of the system, it can be roughly divided into maintenance management, equipment management, warehouse management, and statistical query modules. The maintenance management is divided into the following modules: maintenance application module, maintenance processing module, maintenance dispatch module, maintenance cost registration module, maintenance evaluation module, etc. The equipment warehouse management module is divided into equipment storage, storage, loan, and scrap and others. These functions involve four types of roles, each of which involves more people. Therefore, the system adopts the $\mathrm{B} / \mathrm{S}$ structure, so it can be accessed through the web without downloading the client.

The management of medical equipment involves a wide range of businesses. The functional modules of this system are mainly divided into the following aspects: equipment archive management, contract management, warehouse management, maintenance management, equipment query statistics, etc. Each module is composed of different business functions. Figure 2 shows a block diagram of the medical equipment management system.

3.3. Experimental System Database Design. By comparing the entities included in the system with the database tables, it can be concluded that the system needs to be developed and implemented. The database needs to include equipment supplier tables, equipment tables, equipment type tables, equipment status tables, purchase order tables, purchase schedules, and equipment change application form and user form.

The equipment supplier table is used to store the basic information data of equipment suppliers [36]. The system adds, deletes, and modifies the table through the basic supplier information addition, modification, and deletion functions in the equipment supplier management function module and queries the table through the query function operating. The equipment table is used to store equipment information data. The system uses the supplier equipment information addition, modification, and deletion functions in the equipment supplier management function module to add, delete, and modify the table. Use the query function to query the table, or through equipment information maintenance and statistical query function to operate. The device type table is a data dictionary table of the device type. The system uses the device type information addition, modification, and deletion functions in the system management function module to add, delete, and modify the table and query the table through the query function [37].

\section{Medical Equipment Comprehensive Management System Based on Cloud Computing and Internet of Things}

System development will perform unit testing during the code implementation process and will deploy to the test environment for simulation operation after the development is completed, thereby testing the system as a whole. System testing is a very important link in the software development process, which has a direct impact on the normal operation and maintenance of the software system in the later stages. This section will perform performance testing based on the system functional requirements. Table 1 shows a description of system operation links.

It can be seen from Table 1 that this system relies on the $\mathrm{B} / \mathrm{S}$ framework, so the physical content of the operating environment includes two parts: server and client. The server is installed on the hardware server, including applications, SQL server database, and FineReport service, and the client is an ordinary computer with a browser. The client communicates with the server by sending an HTTP request, and the server communicates with the client by responding to the client's request.

4.1. Medical Equipment Procurement Based on Integrated Management System. If there is no integrated management system for medical equipment based on cloud computing and the Internet of Things, then in the hospital for the purchase of medical equipment, the doctor needs to report to the hospital leader, and the leader will notify the logistics management department after approval, and they will contact the different medical equipment suppliers. In order to reduce the process of medical equipment procurement, this article adds a supplier management module and an equipment procurement management module to the medical equipment integrated management system. Figure 3 shows the statistics on the consumption of medical equipment in the hospital.

It can be seen from Figure 3 that, except for some largescale high-end medical equipment that can be reused for many years, the hospital consumes a large amount of disposable medical equipment every day, including medical 


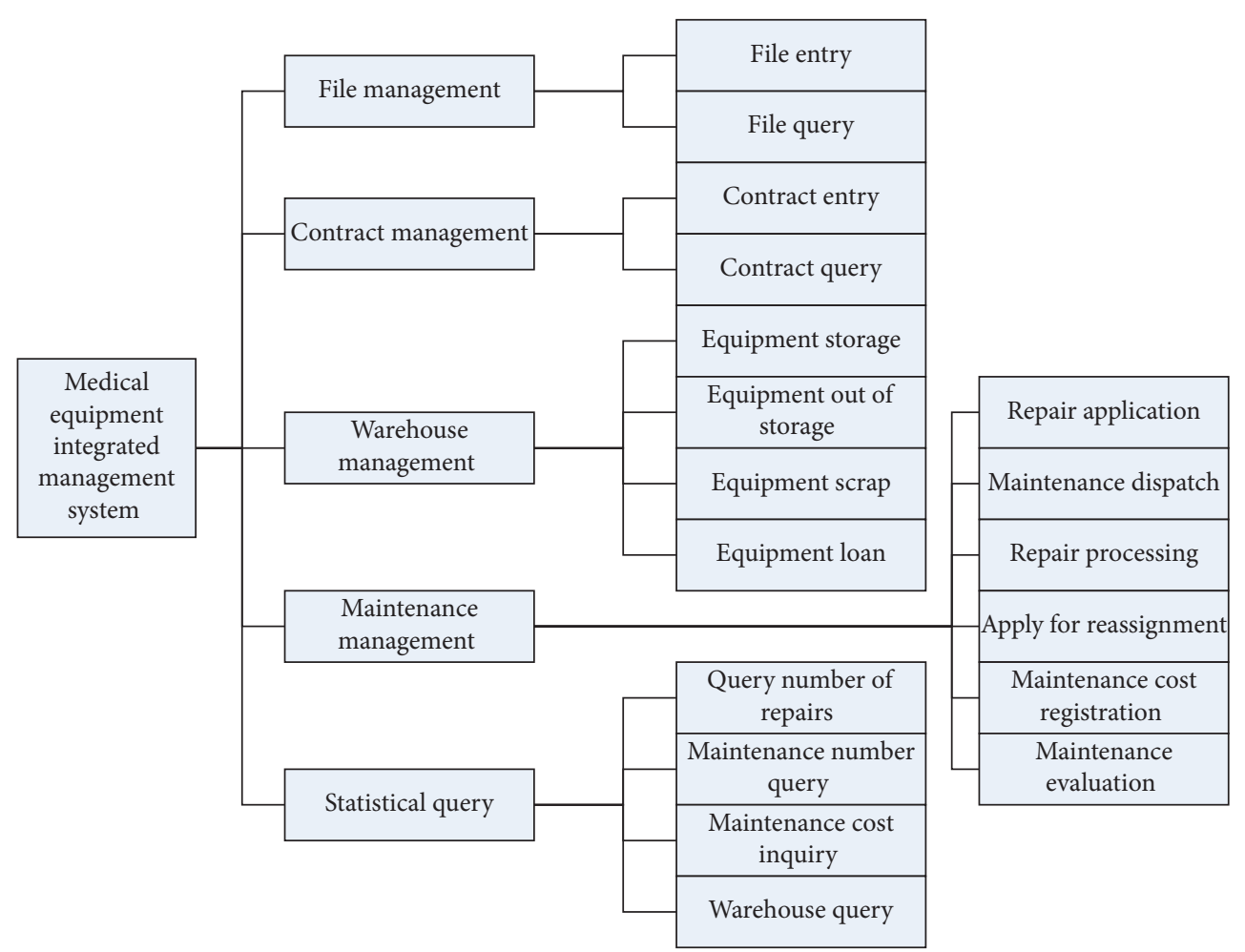

Figure 2: Module structure diagram of medical equipment management system.

TABle 1: System operation description.

\begin{tabular}{lcc}
\hline & Application server & Client \\
\hline \multirow{3}{*}{ Hardware configuration } & Central processing unit: i7 6500U & Central processing unit: i7 6500U \\
& Memory size: DDR4 2400 & Memory size: DDR4 2400 \\
& Hard disk capacity: 1TB 7200 & Hard disk capacity: 1TB 7200 \\
Software configuration & Operating system: Windows 7 & Operating system: Windows 7 \\
& Database: SQL Server 2015 & Report service: FineReport \\
& Application: Device Manager System & Browser: Google Chrome \\
\hline
\end{tabular}

diagnostic equipment, treatment equipment, and various auxiliary equipment. As the inventory data of medical equipment is in a constantly changing state, it is difficult to accurately monitor the consumption of all medical equipment and replenish the inventory in a timely manner if it is manually recorded by management personnel. However, with the assistance of the medical equipment intelligent management system, department leaders and equipment administrators can know the consumption of various equipment at any time and directly purchase products through the system.

Among all kinds of medical equipment, hospitals will stockpile products with a small footprint and a long shelf life on a large scale at a frequency of half a year or even a year. However, those devices with a shorter warranty period and faster version update need to be replenished in real time based on inventory. Figure 4 shows the statistics of medical equipment procurement in the hospital.

It can be seen from Figure 4 that, with the help of the medical equipment intelligent management system, the hospital can realize the purchase of various medical products more intelligently. The equipment administrator only needs to input data in the purchase order information module and the purchase detailed information module, and the background can correctly verify the input data and give a response. It can be seen that the design and implementation of the module can meet the proposed functional requirements.

\subsection{Medical Equipment Maintenance Based on Integrated} Management System. In the medical equipment comprehensive management system, the repair application personnel of the department can enter the system after passing the identity verification and select the equipment repair application business module to fill in the repair application form. The repair personnel are required to enter the repair information such as title, priority, contact person, contact number, repair address, upload fault photos, and repair details. After filling in, click Submit Application for Repair. If all required information has been filled out, it will prompt that the submission is successful. Table 2 shows the maintenance application form in the database, and Figure 5 shows 


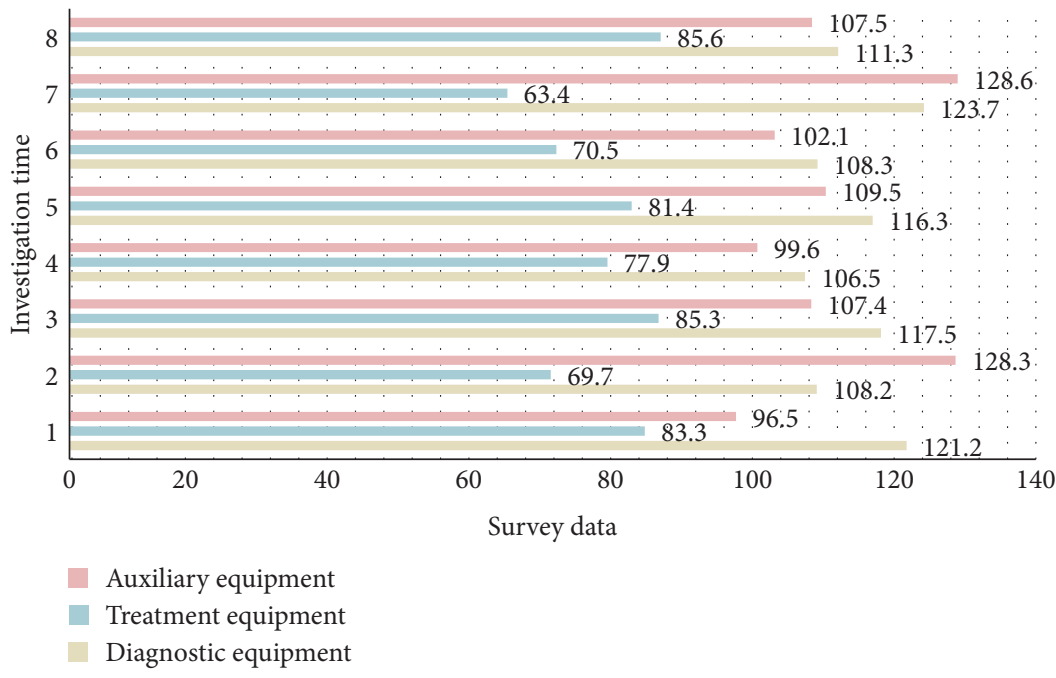

Figure 3: Statistics on the consumption of medical equipment in the hospital.

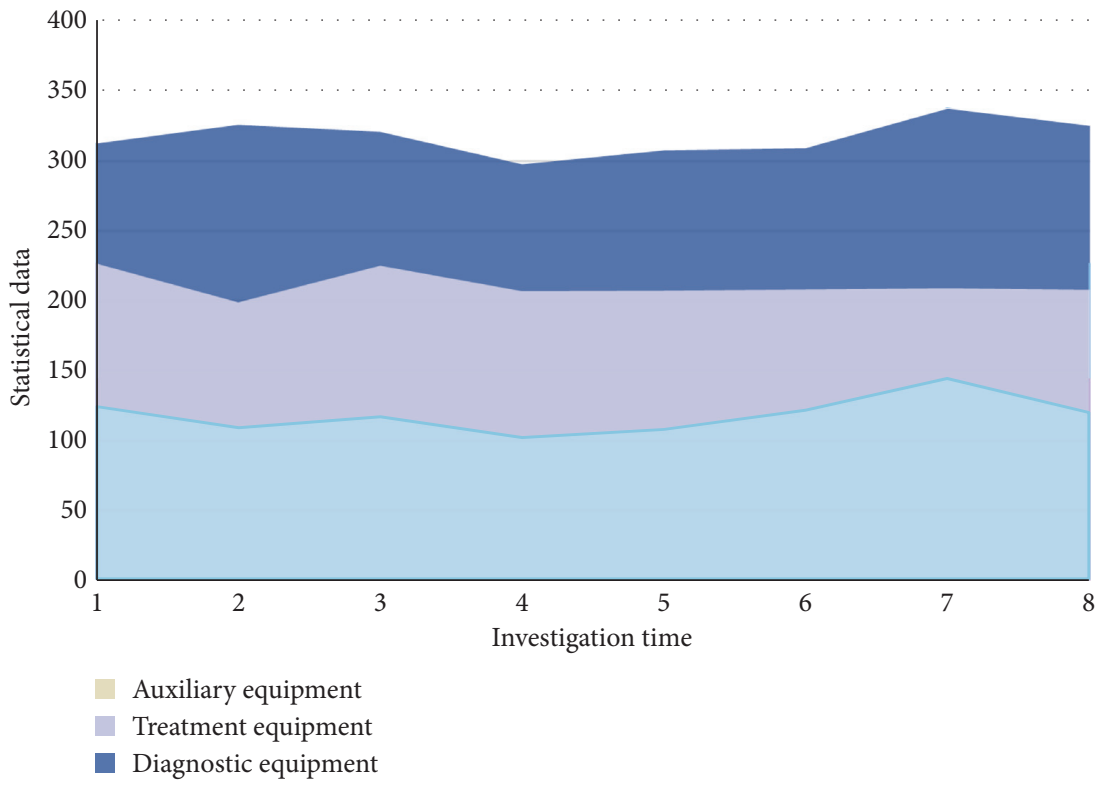

FIGURE 4: Statistics on procurement of medical equipment in the hospital.

TABLE 2: Repair request form in the database.

\begin{tabular}{lcccc}
\hline Field description & Field name & Types & Primary key & Nonempty \\
\hline Repair order number & Service ID & Var char (20) & Yes & Yes \\
Device name & Equipment name & Var char (20) & No & No \\
Device ID & Equipment ID & Var char (20) & No \\
Fault description & Malfunction ms & Var char (20) & No & No \\
Application time & Time & Var char (20) & No & No \\
Applicant & Application admin & Var char (20) & No & No \\
Use department & Application department & Var char (20) & & \\
\hline
\end{tabular}

the equipment repair module work order statistics in the medical equipment integrated management system.

It can be seen from Table 2 and Figure 5 that the hospital leaders and system administrators can clearly grasp the maintenance status of all medical equipment through the equipment management system. The medical equipment maintenance application module is mainly designed for departments. Each department is assigned an administrator who is responsible for the medical equipment maintenance management application and acceptance of the 


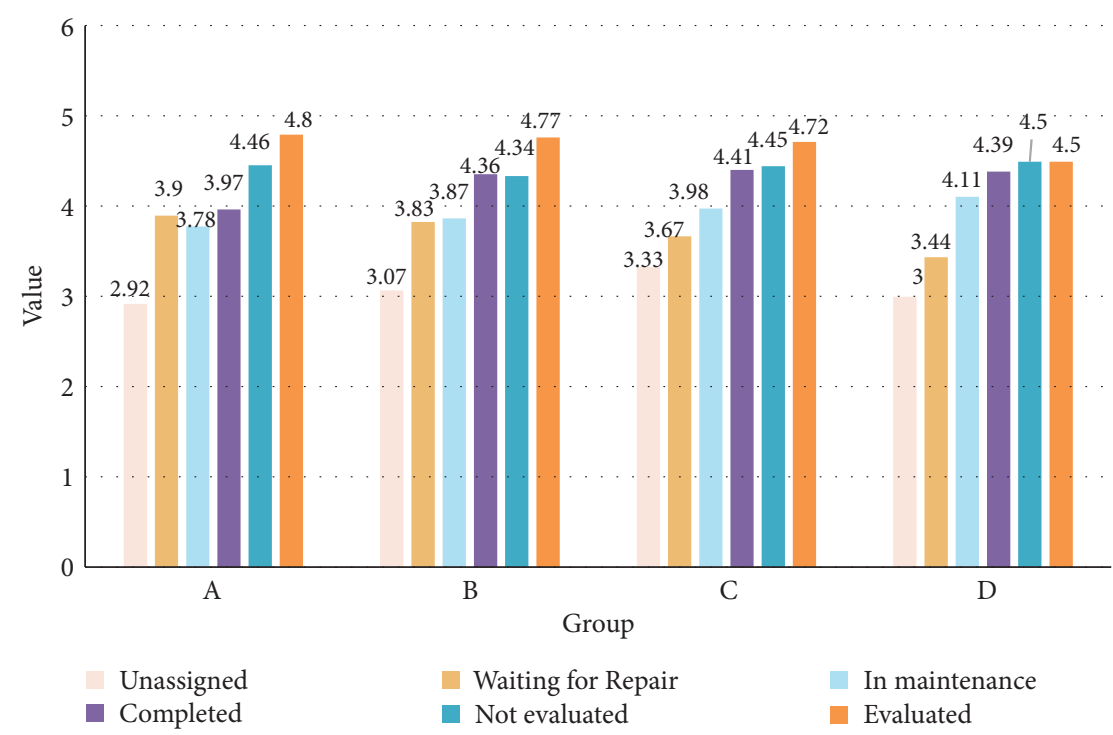

FIGURE 5: Equipment repair module work order statistics.

undergraduate room. After the department administrator registers the system, the system administrator assigns the corresponding authority. After the department administrator logs in to the system, he can inquire about the maintenance of the equipment in the undergraduate room in daily situations, such as the equipment being repaired, repair progress, past repair records, and repair details of these medical equipment.

In order to further grasp the use effect of the medical equipment maintenance template, this paper conducts a system performance test on it. System performance test is divided into two parts: system stress test and system compatibility test. The system stress test is mainly used to test the concurrent operation of multiuser login system. The test system can support multiuser access. During the testing phase, a large number of users cannot be found for simultaneous access system, so this article uses a testing tool for stress testing. The result of the system stress test is shown in Figure 6.

It can be seen from Figure 6 that when there are 100 simultaneous online simulated users, the corresponding time for submitting the equipment maintenance application form is $1228 \mathrm{~ms}$, and the correct rate is $99.8 \%$; when there are 1000 simultaneous online simulated users, the equipment maintenance application form is submitted. The corresponding time is $5123 \mathrm{~ms}$, and the correct rate is $99.4 \%$. Comprehensively observing the engineer's handling of maintenance reports, maintenance equipment inquiries, and information reminders, it can be found that as the number of simulated users increases, the corresponding time of the system is improving, while the accuracy rate has decreased. Taking into account the limited number of people using the system at the same time in the hospital in actual work, maintaining a correct rate of over $99 \%$ is already a relatively good performance for the system.

If we can understand the causes of medical equipment failure, we can further promote the hospital preventive maintenance plan smoothly, so that the equipment has a longer service life. This article takes the monitor as an example and combines various data in the medical equipment management system to explore scientific and reasonable preventive maintenance methods for the monitor. Table 3 and Figure 7 show the breakdown reason classification statistics of the monitor.

It can be seen from Table 3 and Figure 7 that the main reason for the failure of the monitor lies in the accessories. Among them, the blood pressure cuff, the ECG lead wire, and the blood oxygen probe have a relatively high number of failures; among the equipment failures, the number of occurrences is relatively high. It is the blood pressure module, fan failure, and encoder failure. Through data analysis, it is possible to provide reference opinions on the quantity and quality of the purchase of monitor accessories every year, and it is recommended to maintain a corresponding number of vulnerable module accessories for equipment failures, so as to effectively prevent long-term downtime from affecting clinical work and improve equipment utilization. Fan failure is mainly due to fan aging and dust accumulation. For machines with poor heat dissipation, replace the fan and clean up internal dust in time. Encoder failure is the failure of the keys. Replace the encoder with a new one in time for the machine with key problems. Since most of the monitoring consumables are plastic products, they will harden and become unusable if stored for too long.

\subsection{Medical Equipment Management Based on Cloud Com-} puting and Internet of Things. The integrated management of medical equipment is essentially a dynamic task scheduling problem, and the research of task scheduling has always been a hot issue of scientific research in the cloud environment. The problem of cloud computing task scheduling is the process of matching different tasks from different users to cloud computing virtual resources in a reasonable manner through scheduling requirements under certain constraints to meet customer needs. In order to meet the needs of the 


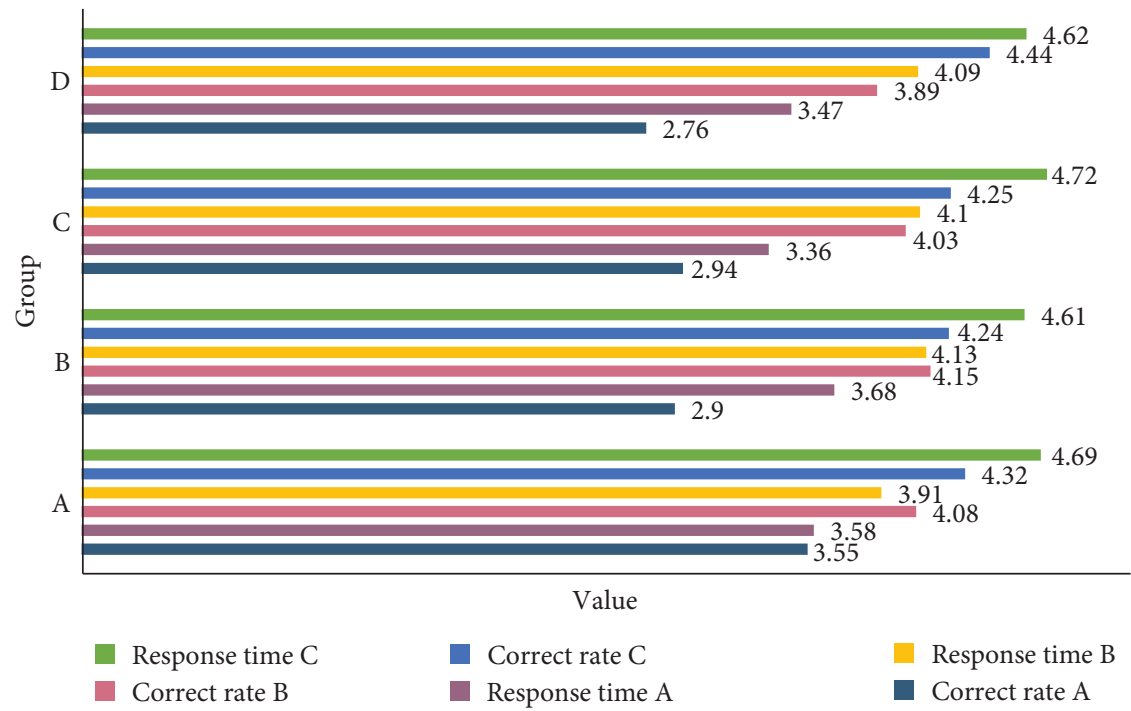

FIgURE 6: Statistical results of system stress test.

TABLE 3: Monitor breakdown reason classification statistics table.

\begin{tabular}{|c|c|c|}
\hline Cause of issue & & Number of failures \\
\hline \multirow{3}{*}{ Accessories failure } & Blood oxygen probe & 162 \\
\hline & ECG lead wire & 130 \\
\hline & Blood pressure cuff & 195 \\
\hline \multirow{9}{*}{ Equipment failure } & Blood oxygen module & 28 \\
\hline & Blood pressure module & 70 \\
\hline & ECG module & 28 \\
\hline & Power board & 11 \\
\hline & Fan & 43 \\
\hline & Display screen & 8 \\
\hline & Keyboard & 12 \\
\hline & Encoder & 46 \\
\hline & Main control board & 9 \\
\hline
\end{tabular}

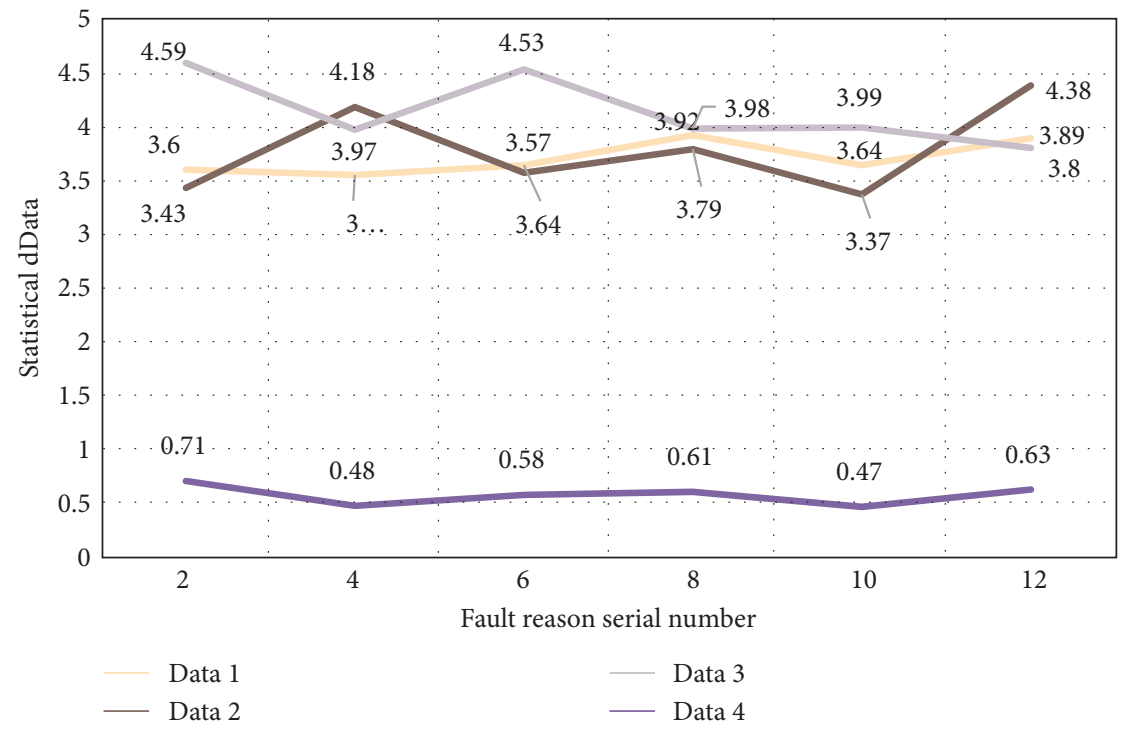

FIGURE 7: Classification statistics chart of monitor failure causes. 


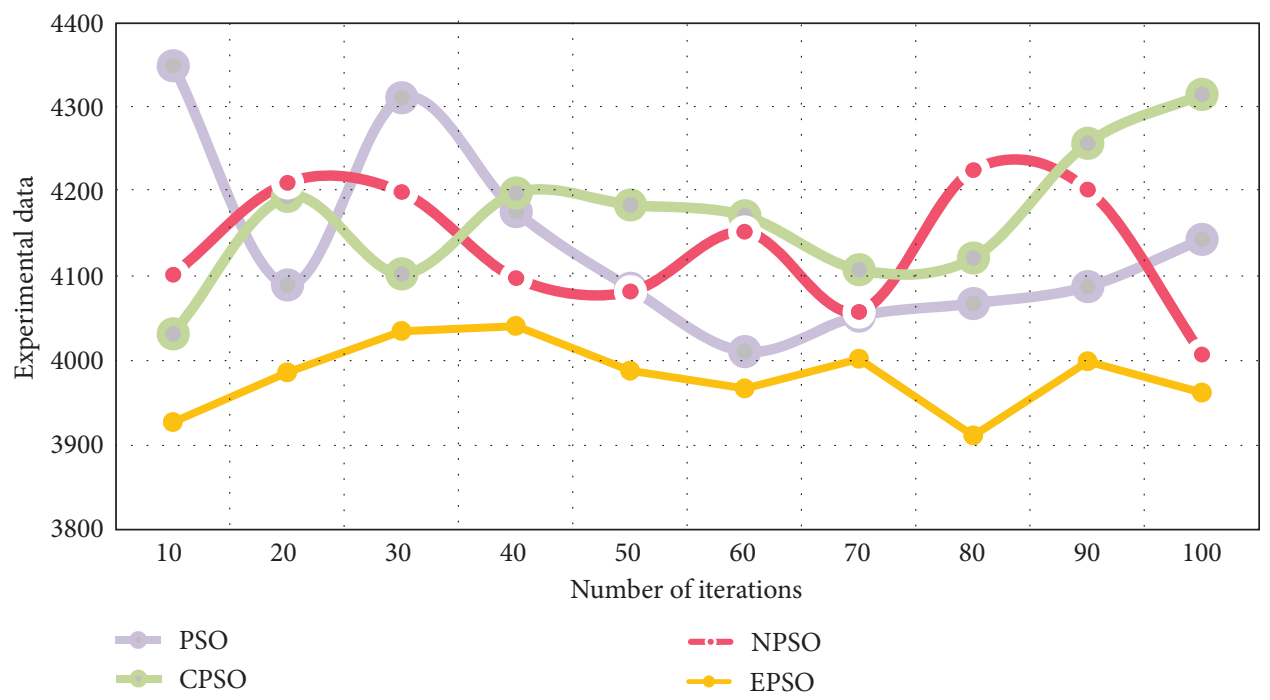

FIgURE 8: Time statistics for particle swarm optimization task optimization.

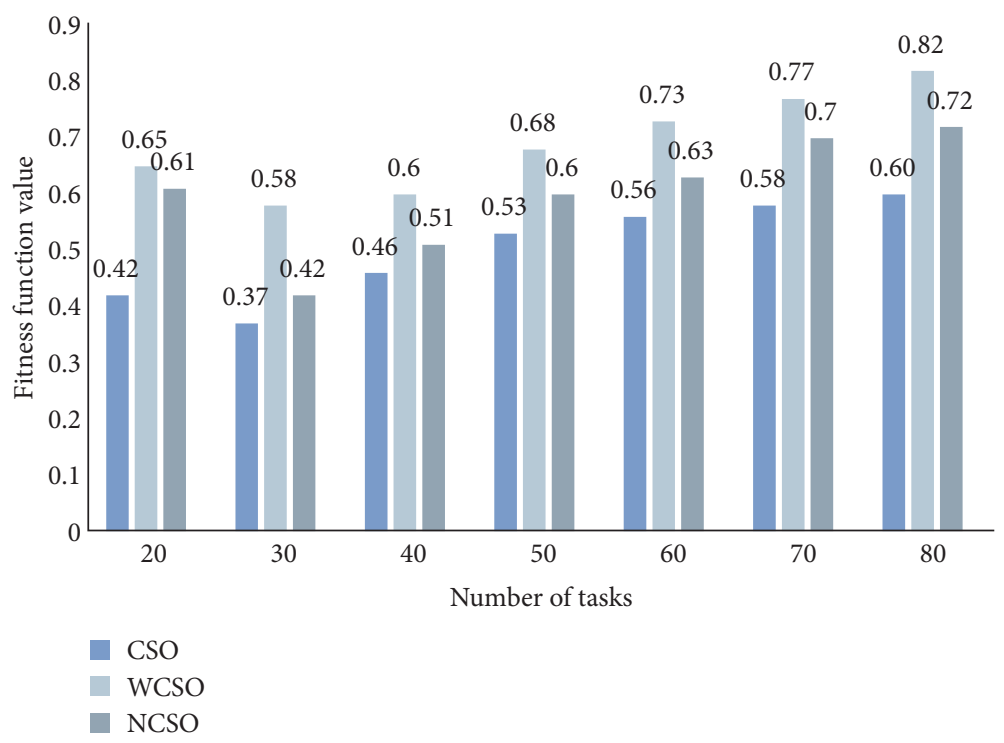

FIGURE 9: Comparison chart of the improved CSO algorithm fitness value.

times, this paper appropriately improves the heuristic intelligent task scheduling algorithm-particle swarm algorithm and chicken swarm optimization algorithm-to improve the accuracy of the scheduling of medical equipment in the system. Figure 8 shows the optimization time statistics of particle swarm optimization when the number of tasks is 50 .

It can be seen from Figure 8 that when the number of tasks is 50 , the task completion time of the particle swarm algorithm will fluctuate differently as the number of iterations changes. Compared with the other three types of particle swarm algorithms, the improved EPSO algorithm in this paper has significantly shorter optimization time. When the number of tasks is low, the gap is relatively small, but as the number of tasks continues to grow, the time efficiency advantage of the improved particle swarm algorithm will become more and more significant. Figure 9 shows a comparison chart of the fitness value of the improved chicken flock algorithm.

It can be seen from Figure 9 that when the number of tasks is the same, the fitness function value of the improved ECSO algorithm in this paper is higher than the original two types of CSO algorithm, which shows that the degree of load balancing is better. When the fitness function value is close to 1 , the longest time it takes for the task to complete is closer to the total time for task completion, and the more balanced the task allocation is when performing scheduling. On the whole, the improvement of the chicken flock algorithm can effectively improve the utilization rate of the system and the efficiency of task execution, so that the system can be better applied to practical problems, and the scientific management and scheduling of medical equipment in the hospital can be realized. 


\section{Conclusions}

This article analyzes the purchase, maintenance, management, and query of medical equipment based on the integrated management system. Through multiple stress tests on the system, the results show that the medical equipment management information system designed and implemented in this paper has excellent effects in terms of system reflection sensitivity, system compatibility, and system stability and fully realizes the initial performance of the prediction. Claim. In this paper, according to the system function modules, the supplier management, equipment purchase management, equipment maintenance management, statistical query, and system management functions were tested in turn. From the results, it can be seen that each module can play a good effect. Due to the full use of the advantages of the Internet of Things and cloud computing task scheduling, the medical equipment management system developed in this paper not only reduces the implementation cost, but also effectively reduces the cost of hospital use. From the perspective of the supplier, it is also beneficial to increase economic benefits.

When scheduling tasks, whether it is time or cost, the more tasks there are, the greater the difference between the scheduling scheme obtained by the improved particle swarm algorithm and the scheduling schemes of the other three algorithms, and the trend of EPSO algorithm change is relatively stable. This is because when the number of tasks is small, each algorithm performs small-scale search, which cannot reflect the advantages of adaptive inertial weight balancing local and global search, and the recognition of individual optimal solutions and global optimal solutions in the particle optimization process relationship. As the number of tasks increases, the inertial weight adaptation and the correlation of random factors have a more significant impact on the particle optimization process, which can better balance the global and local search, thereby obtaining a better scheduling plan. When the fitness function value is close to 1 , the longest time it takes for the task to complete is closer to the total time for task completion and the more balanced the task allocation is when performing scheduling. In the case of the same number of tasks, the fitness function value of the improved ECSO algorithm in this paper is higher than the original two types of CSO algorithm, which shows that the degree of load balancing is better.

In the diagnosis and treatment of diseases, medical equipment has become an indispensable auxiliary means. Whether the medical equipment is advanced or not represents the level of hospital diagnosis and treatment. Therefore, it is very important to carry out effective and scientific management of hospital medical equipment. Judging from the experimental results, the research in this paper has achieved certain results, but limited by various conditions, there are still some shortcomings. In the followup research, more thoughts can be made from the following aspects: (1) Further define the judgment standard of load balancing in task scheduling in the cloud computing environment. (2) In the development process of the medical equipment integrated management system, some modules' functions are not complete enough. (3) It is necessary to propose a multifaceted fitness function for the fusion algorithm according to the requirements of task scheduling. (4) Take advantage of the information age and actively explore a comprehensive management system for medical equipment based on mobile terminals.

\section{Data Availability}

No data were used to support this study.

\section{Conflicts of Interest}

The authors declare that they have no conflicts of interest regarding the publication of this paper.

\section{Acknowledgments}

This work was supported by the Science Research Funding Project JYTJCRW2020089 of Liaoning Provincial Education Department in 2020, Research on Information Sharing of Regional Medical Alliance for Major Diseases Based on Cloud Computing. JYTJCRW2020089

\section{References}

[1] K. G. Srinivasa, B. J. Sowmya, A. Shikhar, R. Utkarsha, and A. Singh, "Data analytics assisted Internet of things towards building intelligent healthcare monitoring systems: iot for healthcare," Journal of Organizational and End User Computing, vol. 30, no. 4, pp. 83-103, 2018.

[2] A. Amerieon, M. Alijanzadeh, and E. Teymourzadeh, "Effective factors on the management of medical equipment maintenance in a military hospital: a qualitative study in Iran," Invertebrate Reproduction and Development, vol. 59, no. 1, pp. 73-84, 2015.

[3] J. M. Ulickey, "One dashboard to rule them all: case study of an integrated facility management system," Energy Engineering, vol. 113, no. 5, pp. 52-63, 2016.

[4] T. Grubljesic, P. S. Coelho, and J. Jaklic, "The shift to socioorganizational drivers of business intelligence and analytics acceptance," Journal of Organizational and End User Computing, vol. 31, no. 2, pp. 37-64, 2019.

[5] Z. Qiang, D. Wen-Yan, and C. Kui, "Discussion on management model of medical equipment maintenance in hospital," China Medical Equipment, vol. 31, no. 5, pp. 1-4, 2016.

[6] S. Namasudra and P. Roy, "PpBAC," Journal of Organizational and End User Computing, vol. 30, no. 4, pp. 14-31, 2018.

[7] X. Chen, L. Jiao, W. Li, and X. Fu, "Efficient multi-user computation offloading for mobile-edge cloud computing," IEEE/ACM Transactions on Networking, vol. 24, no. 5, pp. 2795-2808, 2016.

[8] R. C. Shah and Y. Wang, "Cloud Things Construction - the integration of Internet of things and cloud computing," Future Generation Computer Systems, vol. 56, no. C, pp. 684700, 2016.

[9] Z. Lv and W. Xiu, "Interaction of edge-cloud computing based on SDN and NFV for next generation IoT," IEEE Internet of Things Journal, no. 99, p. 1, 2019.

[10] Z. Xia, X. Wang, L. Zhang et al., "A privacy-preserving and copy-deterrence content-based image retrieval scheme in 
cloud computing," IEEE Transactions on Information Forensics \& Security, vol. 11, no. 11, pp. 2594-2608, 2017.

[11] R. S. Bhadoria and N. S. Chaudhari, "Pragmatic sensory data semantics with service-oriented computing," Journal of Organizational and End User Computing, vol. 31, no. 2, pp. 22-36, 2019.

[12] Q. Yan, F. R. Yu, Q. Gong, and J. Li, "Software-defined networking ( $\mathrm{sdn}$ ) and distributed denial of service (DDoS) attacks in cloud computing environments: a survey, some research issues, and challenges," IEEE Communications Surveys \& Tutorials, vol. 18, no. 1, pp. 602-622, 2016.

[13] C.-S. Chen, W.-Y. Liang, and H.-Y. Hsu, "A cloud computing platform for ERP applications," Applied Soft Computing, vol. 27, no. C, pp. 127-136, 2015.

[14] B. Xu, L. Xu, H. Cai, L. Jiang, Y. Luo, and Y. Gu, "The design of an $\mathrm{m}$-Health monitoring system based on a cloud computing platform," Enterprise Information Systems, vol. 11, no. 1, pp. 17-36, 2017.

[15] Y. Chen, W. Zheng, W. Li, and Y. Huang, "The robustness and sustainability of port logistics systems for emergency supplies from overseas," Journal of Advanced Transportation, vol. 2020, Article ID 8868533, 10 pages, 2020.

[16] D. He and S. Zeadally, "An analysis of RFID authentication schemes for Internet of things in healthcare environment using elliptic curve cryptography," IEEE Internet of Things Journal, vol. 2, no. 1, pp. 72-83, 2015.

[17] S. R. Shanmugham and S. Paramasivam, "Survey on power analysis attacks and its impact on intelligent sensor networks," IET Wireless Sensor Systems, vol. 8, no. 6, pp. 295-304, 2018.

[18] T. Onoue, M. Goto, T. Kobayashi et al., "Randomized controlled trial for assessment of Internet of Things system to guide intensive glucose control in diabetes outpatients: nagoya Health Navigator Study protocol," Nagoya Journal of Medical Science, vol. 79, no. 3, pp. 323-329, 2017.

[19] J. Sun, X. Wu, V. Palade et al., "Random drift particle swarm optimization algorithm: convergence analysis and parameter selection," Machine Learning, vol. 101, no. 1-3, pp. 345-376, 2015.

[20] X. I. Maolong, W. U. Xiaojun, F. Wei et al., "Quantum-behaved particle swarm optimization algorithm with self-renewal mechanism," Computer Engineering and Applications, vol. 2, no. 10, pp. 100-114, 2015.

[21] X. Li and M. Yin, "A particle swarm inspired cuckoo search algorithm for real parameter optimization," Soft Computing, vol. 20, no. 4, pp. 1389-1413, 2016.

[22] A. Rana and D. Sharma, "Mobile ad-hoc clustering using inclusive particle swarm optimization algorithm," International Journal of Electronics and Information Engineering, vol. 8, no. 1, pp. 1-8, 2018.

[23] A. A. A. Esmin, R. A. Coelho, and S. Matwin, "A review on particle swarm optimization algorithm and its variants to clustering high-dimensional data," Artificial Intelligence Review, vol. 44, no. 1, pp. 23-45, 2015.

[24] E. Demirci, O. E. Sahin, M. Ocak, B. Akovali, J. Nematyazar, and L. Kabasakal, "Normal distribution pattern and physiological variants of 68Ga-PSMA-11 PET/CT imaging," Nuclear Medicine Communications, vol. 37, no. 11, pp. 1169-1179, 2016.

[25] N. K. Jain, U. Nangia, and J. Jain, “A review of particle swarm optimization," Journal of the Institution of Engineers, vol. 99, no. 4, pp. 1-5, 2018.

[26] Y. Tang and X. Guan, "Parameter estimation for time-delay chaotic system by particle swarm optimization," Chaos Solitons and Fractals, vol. 40, no. 3, pp. 1391-1398, 2017.
[27] A. Francis Saviour Devaraj, M. Elhoseny, S. Dhanasekaran, E. Laxmi Lydia, and K. Shankar, "Hybridization of firefly and improved multi-objective particle swarm optimization algorithm for energy efficient load balancing in cloud computing environments," Journal of Parallel and Distributed Computing, 2020, In Press.

[28] V. Viswanathan and I. Krishnamurthi, "Finding relevant semantic association paths using semantic ant colony optimization algorithm," Soft Computing, vol. 19, no. 1, pp. 251-260, 2015.

[29] Y. Huan, "Image edge detection based on ant colony optimization algorithm," International Journal of Advanced Pervasive and Ubiquitous Computing, vol. 8, no. 1, pp. 1-12, 2016.

[30] T. Liao and Y. Yu, "Mixed variable and any time optimization oriented ant colony optimization algorithm," Systems Engineering and Electronics, vol. 39, no. 3, pp. 675-680, 2017.

[31] M.-Y. Cheng, D. Prayogo, Y.-W. Wu, and M. M. Lukito, “A Hybrid Harmony Search algorithm for discrete sizing optimization of truss structure," Automation in Construction, vol. 69, no. SEP, pp. 21-33, 2016.

[32] H. Zhang, S. Qu, H. Li, J. Luo, and W. Xu, "A moving shadow elimination method based on fusion of multi-feature," IEEE Access, vol. 8, pp. 63971-63982, 2020.

[33] B. González, F. Valdez, P. Melin, and G. Prado-Arechiga, "Fuzzy logic in the gravitational search algorithm for the optimization of modular neural networks in pattern recognition," Expert Systems with Applications, vol. 42, no. 14, pp. 5839-5847, 2015.

[34] G. Zhang and Y. Li, "A memetic algorithm for global optimization of multimodal nonseparable problems," IEEE Transactions on Cybernetics, vol. 46, no. 6, pp. 1375-1387, 2016.

[35] L. Yin, W. Pan, J. Kuang, and M. Zhuang, "Application of bootstrap-DEA with fuzzy computing in performance evaluation of forklift leasing supplier," IEEE Access, vol. 8, pp. 66095-66104, 2020.

[36] R. Parada, J. Melià-Seguí, and R. Pous, "Anomaly detection using rfid-based information management in an iot context," Journal of Organizational and End User Computing, vol. 30, no. 3, pp. 1-23, 2018.

[37] Z. Lv and N. Kumar, "Software defined solutions for sensors in 6G/IoE," Computer Communications, vol. 153, pp. 42-47, 2020. 